\title{
Conf-940580--24
}

UCRL-JC-115909

PREPRINT

\section{Techniques and Results of Tokamak-Edge Simulation}

\author{
G.R. Smith \\ P.N. Brown \\ R.B. Campbell \\ D.A. Knoll \\ P.R. McHugh \\ M.E. Rensink \\ T.D. Rognlien
}

This paper was prepared for submittal to

11th Intl. Conf. on Plasma Surface Interactions in Controlled Fusion Devices

May 23-27, 1994

Mito-shi, Ibaraki-ken, Japan

May 20.1994

This is a preprint of a paper intended for publication in a journal or proceedings. Since changes may be made before publication, this preprint is made available with the understanding that it will not be cited or reproduced without the permission of the author.
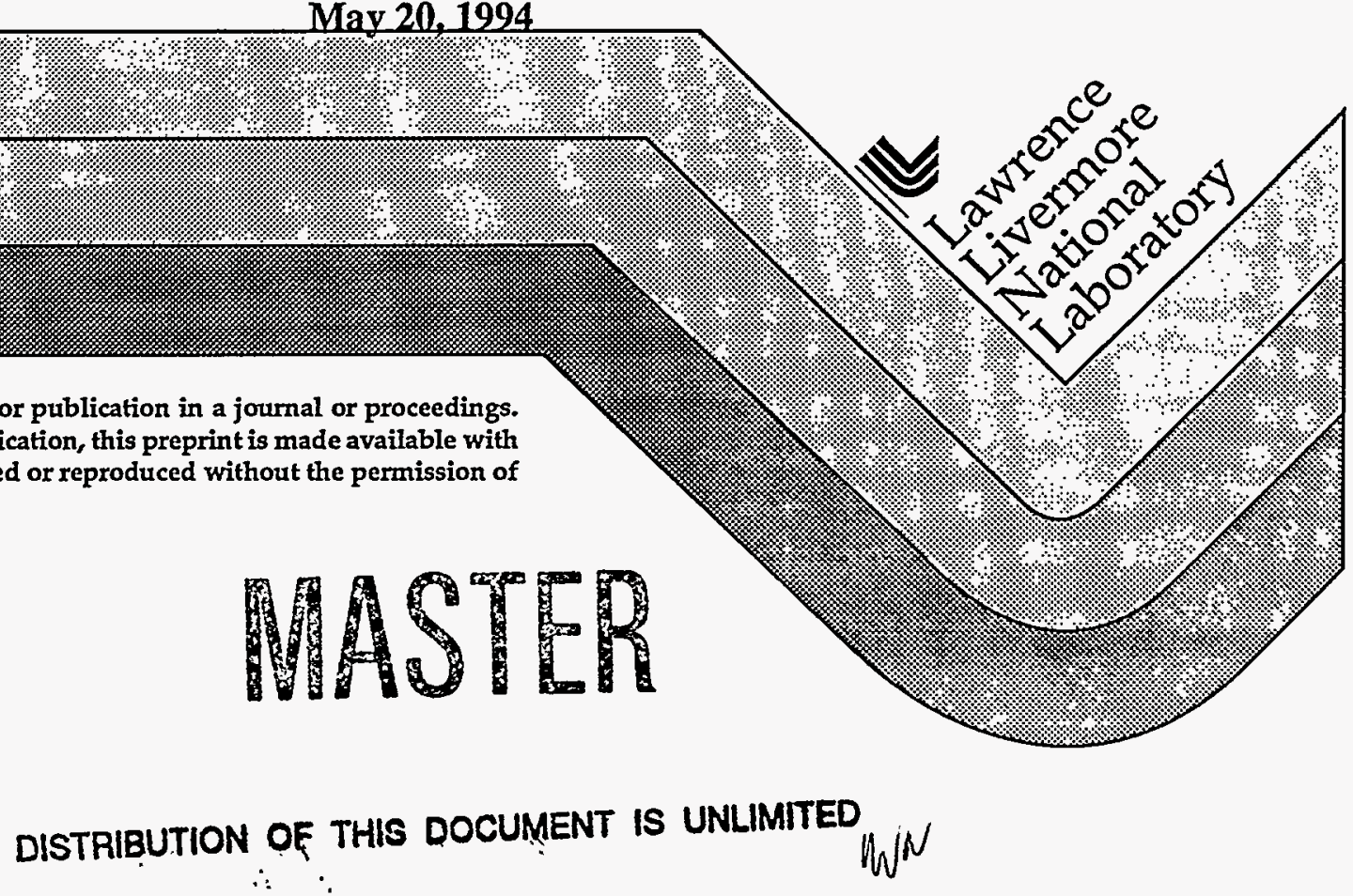


\section{DISCIAIMER}

This document was prepared as an account of work sponsored by an agency of the United States Government. Neither the United States Government nor the University of Californiz nor any of their employecs, makes any warranty, express or implied, or assumes any tegal liability or responsibility for the accuracy; completeness, or usefulness of any information, apparatus, product, or process disclosed, or represents that its use would not infringe privately owned rights. Reference herein to any specific commercial products, process, or service by trade riame. trademark, manufacturer, or otherwise, does not necessarily constitute or imply its endorsement, recommeadation, or favoring by the United States Government or the University of California. The vieus and opinions of authors expressed herein do not necessarily state or reflect those of the United States Government or the University of California, and shall not be used for advertising or product endorsement purposes.

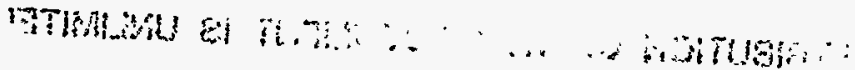




\section{DISCLAIMER}

Portions of this document may be illegible in electronic image products. Images are produced from the best available original document. 


\title{
Techniques and Results of Tokamak-Edge Simulation
}

\author{
G.R. Smith, P.N. Brown, R.B. Campbell*, D.A. Knoll ${ }^{\dagger}$, P.R. McHugh ${ }^{\dagger}$, \\ M.E. Rensink, and T.D. Rognlien \\ Lawrence Livermore National Laboratory, University of California, \\ Livermore, California 94551, USA
}

This paper describes recent development of the UEDGE code in three important areas. (1) Non-orthogonal grids allow accurate treatment of experimental geometries in which divertor plates intersect flux surfaces at oblique angles. (2) Radiating impurities are included by means of one or more continuity equations that describe transport and sources and sinks due to ionization and recombination processes. (3) Advanced iterative methods that reduce storage and execution time allow us to find fully converged solutions of larger problems (i.e., finer grids). Sample calculations are presented to illustrate these developments.

DISTRIBUTION OF THIS DOCUMENT IS UNLIMITED WV $^{\prime}$

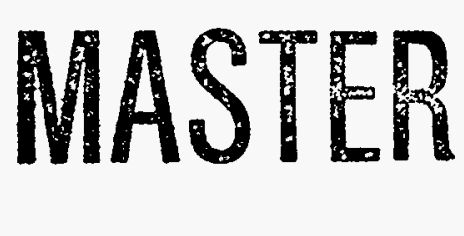

${ }^{*}$ Sandia National Laboratories - New Mexico, Albuquerque, NM 87185, USA

IIdaho National Engineering Laboratory, Idaho Falls, ID 83415, USA 


\section{Introduction}

Fluid models of the tokamak edge plasma are developing rapidly through the efforts of several groups and are reviewed in Ref. [1]. The UEDGE simulation code is used to find equilibrium and time-dependent solutions of a two-dimensional fluid model of the tokamak edge as described in Ref. [2]. All of the 2-D models solve in the poloidal plane and assume classical parallel (and thus poloidal) transport and anomalous radial transport. The solution algorithms do differ, and the UEDGE code utilizes a fully implicit method with a numerical Jacobian. The need to interpret experiments and design new divertors has led to ever more realistic models. This paper describes recent improvements made to UEDGE and presents some new results.

\section{Improvements in the UEDGE Simulation Code}

\subsection{Non-Orthogonal Grid}

Divertor plates and other material surfaces that interact with the edge plasma are generally not orthogonal to the magnetic flux surfaces. To treat such situations effectively, we use a non-orthogonal grid that has one set of grid lines parallel to flux surfaces and the other set locally parallel to the plates or material surfaces. Keeping one set of grid lines parallel to flux surfaces minimizes finite-difference errors in transport across flux surfaces caused by the much more rapid parallel transport. The numerical task of forming the finite-volume equations to be solved requires finitedifference estimation of the fluxes passing through the faces of the volumes represented by each grid cell. As illustrated in Fig. 1(a), the required fluxes are $\Gamma_{y}$ in the "radial" direction perpendicular to magnetic flux surfaces $(y=$ constant $)$ and $\Gamma_{n}$ in the direction normal to the other cell faces. At the center of a cell face, that normal makes an angle $\alpha$ with the magnetic flux surface, and the flux there can be calculated from components $\Gamma_{x}$ and $\Gamma_{y}$ in the two coordinate directions

$$
\Gamma_{n}=\Gamma_{y} \sin \alpha+\Gamma_{x} \cos \alpha
$$

Those flux components are determined by:convective flow velocity components $v_{y}$ and $v_{x}$, by elements $D_{y}$ and $D_{\dot{x}}$ of the diagonal but anisotropic, $2 \mathrm{D}$ diffusion tensor, and by gradients $\nabla_{y} \phi$ and $\nabla_{x} \phi$ of any quantity $\phi(x, y)$ (e.g., particle density) whose flux is desired

$$
\begin{aligned}
& \Gamma_{y}=v_{y}-D_{y} \nabla_{y} \phi \\
& \Gamma_{x}=v_{x}-D_{x} \nabla_{x} \phi .
\end{aligned}
$$


Cross-field drifts can be straightforwardly added by including off-diagonal components of the diffusion tensor. At the face where $\Gamma_{n}$ is required, the radial gradient $\nabla_{y} \phi$ is calculated from the normal and poloidal gradients using

$$
\nabla_{y} \phi=\left(\nabla_{n} \phi-\cos \alpha \nabla_{x} \phi\right) / \sin \alpha,
$$

as indicated in Fig. 1(b). Quantities $v_{y}, \nabla_{n} \phi$, and $\nabla_{x} \phi$ are computed by appropriate linear averaging and differencing of values known at the four grid points nearest the center of the face at which the flux is required. The poloidal velocity $v_{x}$ is known on a grid that is staggered a half cell poloidally from the grid for other quantities, so $v_{x}$ is immediately available for calculation of $\Gamma_{x}$. This procedure results in a 9-point stencil that fits directly into our existing, numerical solution algorithm:

In Fig. 2 we show an example of a non-orthogonal grid used for calculations described in Sec. 3. The grid is distorted to accommodate baffles that are intended to facilitate the pumping of gas and impurities by impeding their.flow away from the divertor plates.

\subsection{Impurities}

The average-ion and multi-charge impurity models developed previously and described in Refs. [3] and [4] have been incorporated into UEDGE. Continuity equations are added to describe the spatial variation of the impurities; one equation is added for the average-ion model, and an equation for each charge state is added for the multicharge impurity model. Ionization and recombination are included. The velocities normal to the flux surfaces are described by the same anomalous diffusion process as for the hydrogenic species. In the poloidal direction, the velocity is obtained from parallel momentum balance neglecting inertia and viscosity; interspecies friction is balanced against the parallel force caused by pressure, thermal, and electric potential gradients. The impurity poloidal velocity, $u_{x z}$, is thus obtained from

$$
\left(u_{x z}-u_{x h}\right) m_{i} n_{i} \nu_{h z}=\left(\frac{B_{x}}{B}\right)^{2}\left(-\frac{\partial P_{z}}{\partial x}+\alpha_{z} n_{z} \frac{\partial T_{e}}{\partial x}+\beta_{z} n_{z} \frac{\partial T_{i}}{\partial x}-Z_{e n_{z}} \frac{\partial \Phi}{\partial x}\right)
$$

where the various terms are $u_{x h}$, hydrogenic velocity; $\nu_{h z}$, interspecies friction rate; $B_{x} / B$, ratio of poloidal to total magnetic field; $P_{z}$, impurity pressure; $T_{e, i}$, electron and ion temperatures; and $\Phi$, electrostatic potential. The $\alpha$ and $\beta$ are numerical coefficients [4].

The average-ion and multi-charge impurity models are available as alternatives to the simple fixed-fraction model used previously [5]. An arbitrary distribution of impurity density can be specified in the fixed-fraction model, but that distribution 
is not required to satisfy any physical equations. For the average-ion and multicharge impurity models, the impurity density distribution is calculated along with hydrogenic-ion density and poloidal flow velocity, electron and ion temperature, and gas density, during iterations to an equilibrium solution.

\subsection{Advanced Iterative Solution Methods}

Our fully implicit formulation of the fluid equations gives us access to the strong convergence properties of Newton's method for the entire finite-differenced equation set. For fine grids the associated Jacobian matrix can require significant computational resources (large storage and long inversion times) [2]. Newton-Krylov methods allow reduction in the storage and time requirements, because an approximate factorization of the Jacobian matrix is sufficient and the sparsity of the Jacobian and its factors can be exploited [6]. Furthermore, the approximation involved in the factorization process can be improved considerably by reordering the rows and columns of the Jacobian matrix [7].

In Fig. 3 we illustrate the strong convergence and the benefits of reordering during Newton-Krylov iteration of a problem described by a total of 5518 equations ( 5 equations in each cell of a $62 \times 18$ grid). The problem being modeled here is gas puffing in the DIII-D tokamak as described in Ref. [8]. The Jacobian is recalculated only every fifth iteration for added efficiency. Three different approximate factorization methods are compared. The first method (curves $A$ in the figure) stores only the matrix elements of the factors $L$ and $U$ that occur in the diagonals that had non-zero elements in the Jacobian. The second method (curves B) does no reordering but allows as many as 100 additional non-zero elements in each row of $L$ and $U$ compared to the number of non-zero elements in the Jacobian. The third method (curves C) does reordering but imposes a tighter limit (35) on the "fill-in" parameter, the number of additional non-zero elements in each row of $L$.and $U$. Part (a) of Fig. 3 shows the performance of Newton-Krylov iteration in reducing the norm of the residuals after an equilibrium solution was perturbed by changing a boundary condition, $T_{e}$ at the coreedge boundary, from 200 to $250 \mathrm{eV}$. Curve A does not show the strong convergence of the other two methods. Curves $\mathrm{B}$ and $\mathrm{C}$ show similarly good performance in reducing the residuals. However, Fig. 3(b) shows that many fewer evaluations of the residual (right-hand-side) function are required with reordering than with either of the other methods. Reduction in the number of residual evaluations leads to a total execution time that is shorter by a factor of about two.

We also take advantage of advanced factorization methods during time-dependent relaxation towards equilibrium, which is of great utility in practice because of its 
greater radius of convergence than Newton methods.

\section{Results with a Non-Orthogonal Grid}

The effect of a non-orthogonal grid is illustrated for a case corresponding to the proposed radiative divertor design for DIII-D [9]. The grid for the lower half of this double null configuration is shown in Fig. 2. In the radial-like direction, magnetic flux surfaces are used, whereas in the poloidal direction, the grid is distorted from orthogonality to conform to the outer baffle and the shaped inner plate surface. The $\mathrm{X}$ 's show the outline of the orthogonal grid used for a comparison calculation that omits the baffle.

We consider a case where the electron and ion temperatures $\left(T_{e}\right.$ and $\left.T_{i}\right)$ on the core boundary are $175 \mathrm{eV}$ and the boundary density is $3 \times 10^{19} \mathrm{~m}^{-3}$. The anomalous radial diffusion coefficients are $D=0.25 \mathrm{~m}^{2} / \mathrm{s}, \chi_{e}=1: 5 \mathrm{~m}^{2} / \mathrm{s}$, and $\chi_{i}=1.0 \mathrm{~m}^{2} / \mathrm{s}$. The resulting power crossing the separatrix is $8: 8 \mathrm{MW}$, with half going to the lower portion being simulated here. The particle recycling coefficient on the divertor plates is set to 1.0. We use these parameters for two calculations, one with an orthogonal grid, but no baffle, and one with the non-orthogonal grid and baffle. The non-orthogonal grid does not degrade the convergence properties of the numerical algorithm.

One of the goals of the divertor design is to prevent gas from escaping the plate region and fueling the core. While the outer baffle is planned to minimize escaping gas, there is the concern that recycling off the baffle itself would create excess gas. Our non-orthogonal grid calculations show that the net effect of the baffle is to reduce the plasma and gas density on the outer flux surfaces while increasing these densities near the separatrix. The results are shown in Fig. 4(a) for the ion density; the gas density profiles are very nearly the same. The profiles are plotted versus the normalized flux . $\psi_{n}$, with unity corresponding to the separatrix. The non-orthogonal grid begins to rise from the horizontal for $\psi_{n}=1.01$, just where the density peaks. The second effect of the baffle is to increase $T_{e}$ along it, as shown in Fig. 4(b). This is caused by the poloidal distance to the baffle being shorter than to the flat plate, so there is less thermal insulation.

\section{Results with the Average-Impurity-Ion Model}

The average-ion model is illustrated with a calculation corresponding again to the DIII-D tokamak. The MHD equilibrium is the same as that used for Fig. 2 and corresponds to the gas-puffing case in Ref. [8]. However, to highlight the impurities, we do not gas puff here and reduce the recycling coefficient to the low value of 0.8 . 
The core-boundary values are $T_{e, i}=100 \mathrm{eV}$ and $n_{i}=2 \times 10^{19} \mathrm{~m}^{-3}$. The 200-Amp source of the carbon impurities is a uniform flux distributed around the outer-wall boundary.

The resulting contours of carbon radiation in the divertor region are shown in Fig. 5. The total power crossing the separatrix is $2 \mathrm{MW}$, and $0.4 \mathrm{MW}$ is radiated by the carbon; the hydrogenic radiation is only $0.14 \mathrm{MW}$ for this low-recycling case. Note that the largest radiation is on the inner divertor leg, resulting in the lowest $T_{e}$ there. This is qualitatively similar to experimental observations, but this initial illustrative calculation needs to be extended to higher recycling and a more detailed carbon source from sputtering.

\section{Summary}

We have highlighted three improvements to the UEDGE code: a non-orthogonal grid that allows curved boundaries, impurity transport models that utilize parallel force balance and anomalous radial diffusion, and advanced iterative techniques for efficient approximate solutions to the Jacobian matrix. Each of these improvements has been illustrated by an example. We have just begun to exercise the impurity routines and plan to focus on refining this capability.

\section{Acknowledgments}

We gratefully acknowledge discussions with R.H. Cohen, M.E. Fenstermacher, A.C. Hindmarsh, and G.D. Porter.

This work was performed under the auspices of the U.S. Department of Energy by Lawrence Livermore National Laboratory under contract No. W-7405-Eng48 , by Sandia National Laboratories - New Mexico under contract No. DE-AC0476DP00789, and by Idaho National Engineering Laboratory under contract No. DEAC07-76ID01570. 


\section{References}

[1] REITER, D., J. Nucl. Mat. 196-198 (1992) 80.

[2] ROGNLIEN, T. D., MILOVICH, J. L., RENSINK, M. E., and PORTER, G. D., J. Nucl. Materials 196-198 (1992) 347.

[3] KNOLL, D. A., CAMPBELL, R. B., and MCHUGH, P. R., Contrib. Plasma Phys. 34 (1994) 386.

[4] KEILHACKER, M., SIMONINI, R., TARONI, A., and WATKINS, M. L., Nucl. Fusion 31 (1991) 535.

[5] ALLEN, S. L., RENSINK, M. E., HILL, D. N., et al., J. Nucl. Materials 196-198 (1992) 804.

[6] BROWN, P. N. and SAAD; Y.; SIAM J. Sci. Stat. Comput. 11 (1990) 450.

[7] SAAD, Y., private communication (1993).

[8] .ROGNLIEN, T. D., BROWN, P. N., CAMPBELL, R. B., et al., Contrib. Plasma Phys. 34 (1994) 362.

[9] ALLEN, S. L., this conference. 


\section{Figures}

Fig. 1. Geometry for calculation of fluxes on a non-orthogonal grid. (a) Grid lines bounding a typical cell, grid points at cell centers, and fluxes $\Gamma_{y}$ and $\Gamma_{n}$ perpendicular to two faces of the typical cell. The directions along $(x)$ and perpendicular to $(y)$ flux surfaces are indicated. (b) For the slanted face at which $\Gamma_{n}$ is required, the normal gradient $\nabla_{n} \phi$ of a quantity $\phi(x, y)$ and its components $\nabla_{x} \phi$ and $\nabla_{y} \phi$ are shown.

Fig. 2. The region near the X-point of a proposed configuration for the DIII-D experiment at General Atomics. Solid lines show the grid, which is distorted to conform at the poloidal grid boundaries to material surfaces that serve as baffles. Dashed lines show the edges of the baffles and the entrances to the pumping ducts. The X's indicate the location of the boundary of the orthogonal grid that is used for comparisons with the non-orthogonal grid in Sec. 3.

Fig. 3. Performance during Newton-Krylov iterations of three solution methods. for the edge-plasma equations. See the text in Sec. 2.3 for a description of the methods. (a) Reduction of the norm of the residuals as a function of iteration number. (b) Cumulative number of evaluations of the residuals as a function of iteration number.

Fig. 4. Comparison of results computed with orthogonal and non-orthogonal grids. (a) Ion density in $\mathrm{m}^{-3}$ plotted versus normalized poloidal flux, $\psi_{n}$, on the outer divertor plate for the case in Fig. 2; solid line, orthogonal grid, and dashed line, non-orthogonal grid with baffle. The separatrix has $\psi_{n}=1.0$. (b) The corresponding electron temperature profiles.

Fig. 5. Carbon radiation contours for the average-ion model obtained for a DIII-D simulation with carbon injected around the outer wall. Numbers on the contours are multiples of $0.1 \mathrm{MW} \mathrm{m}^{-3}$. 

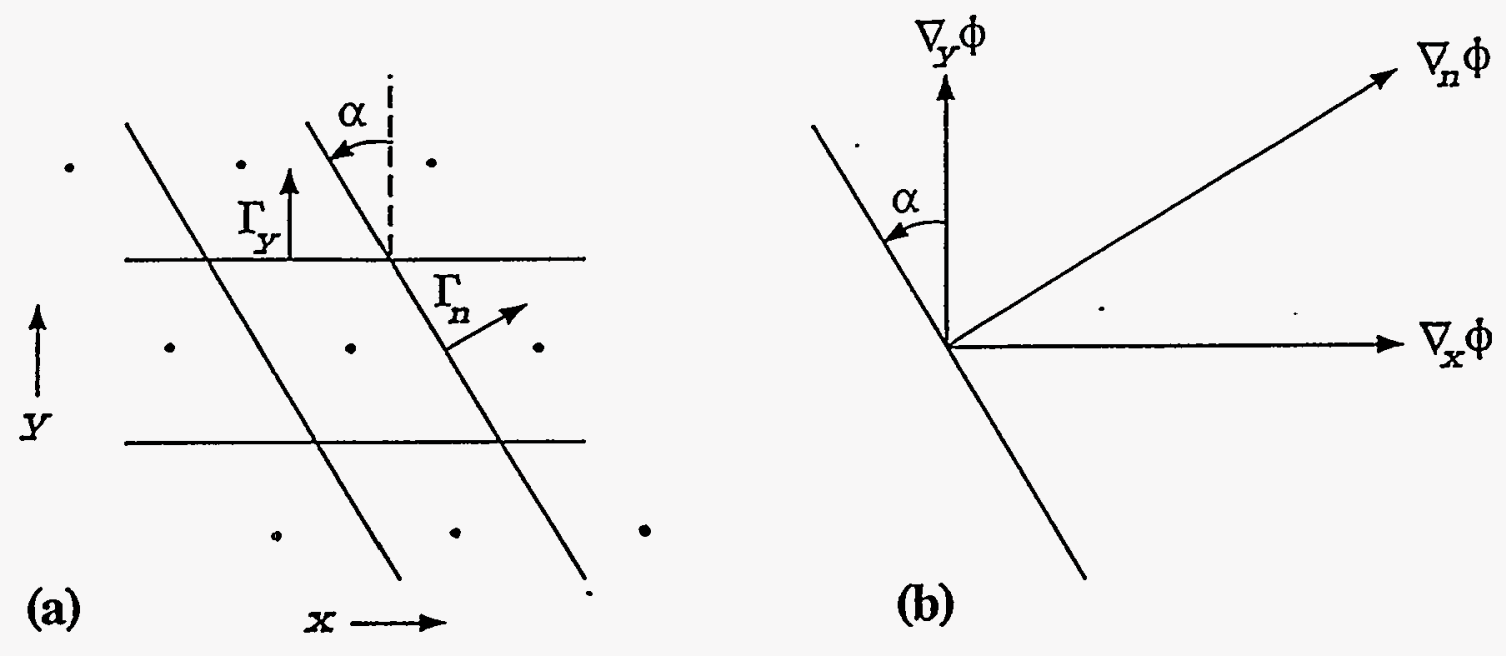

Fig. I 


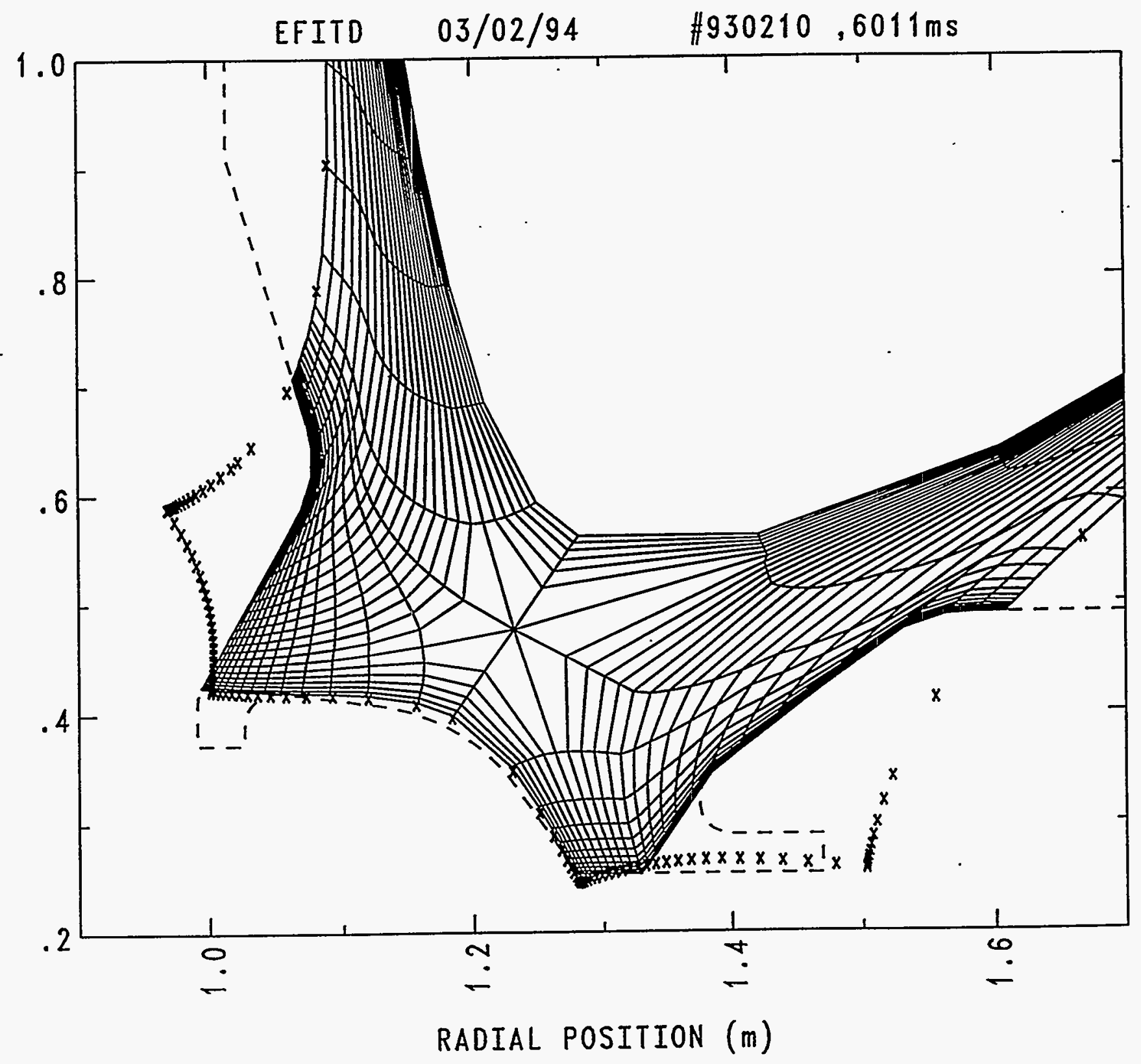

Fig. 2 

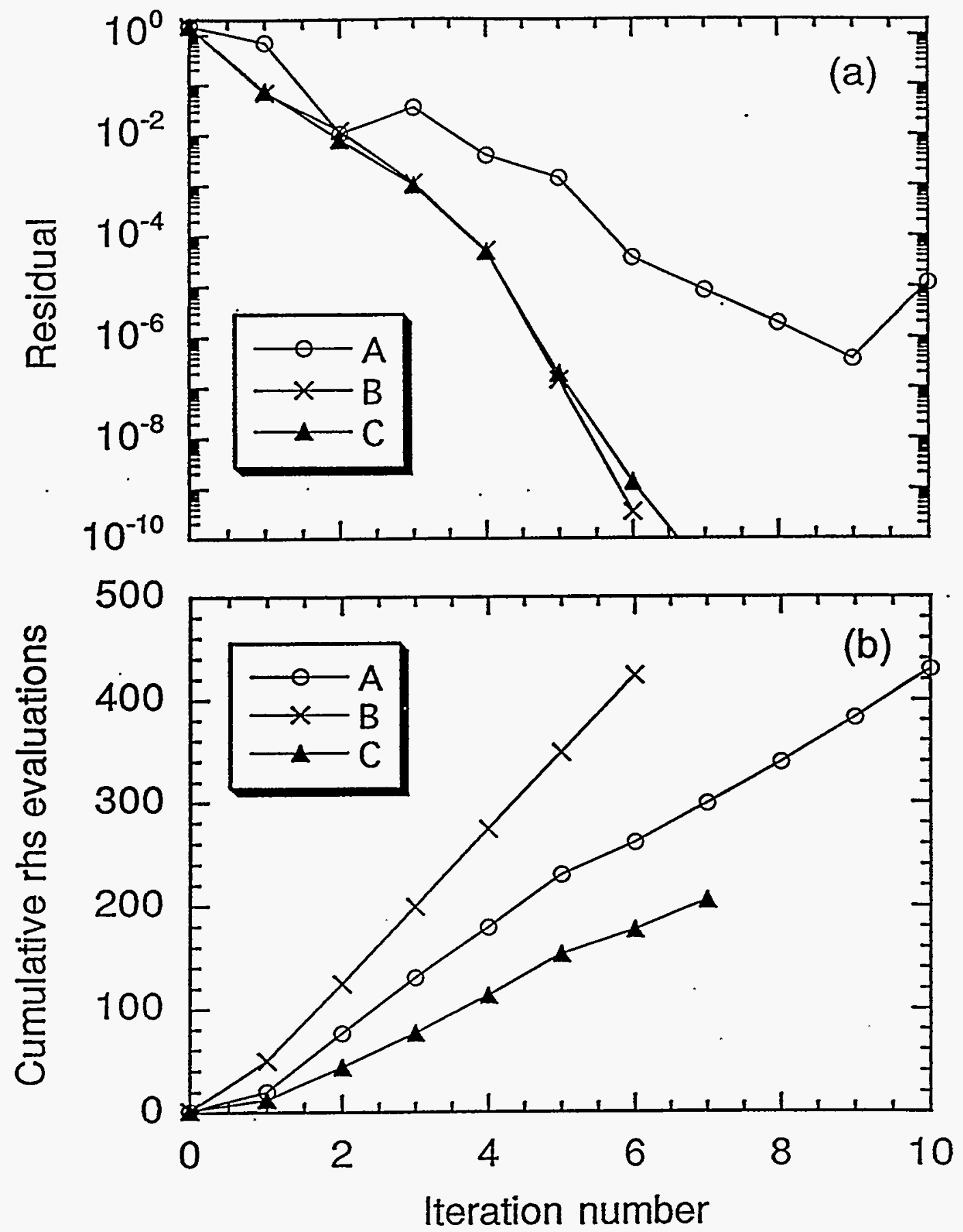

Fig. 3 

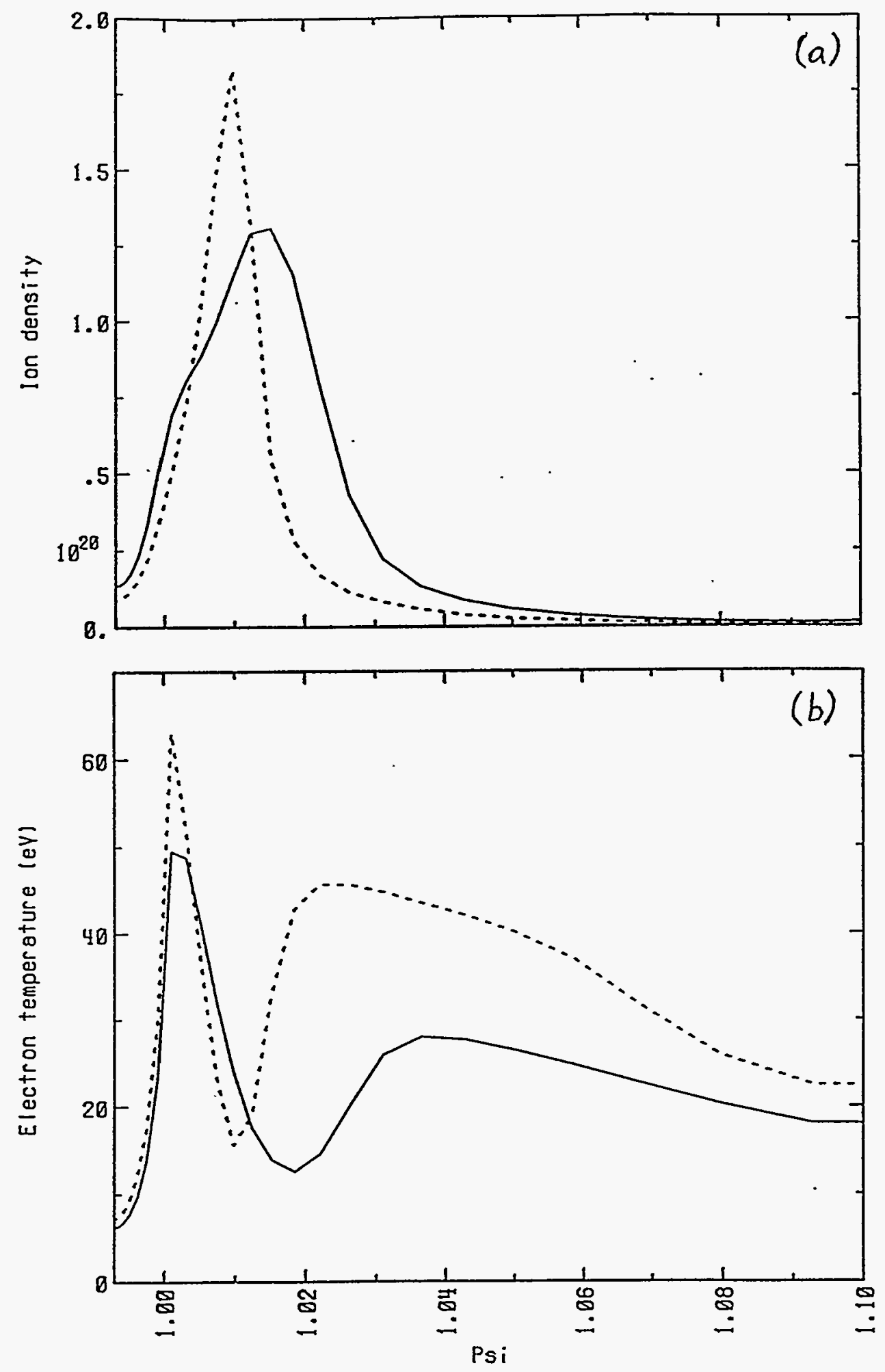

Fig. 4 


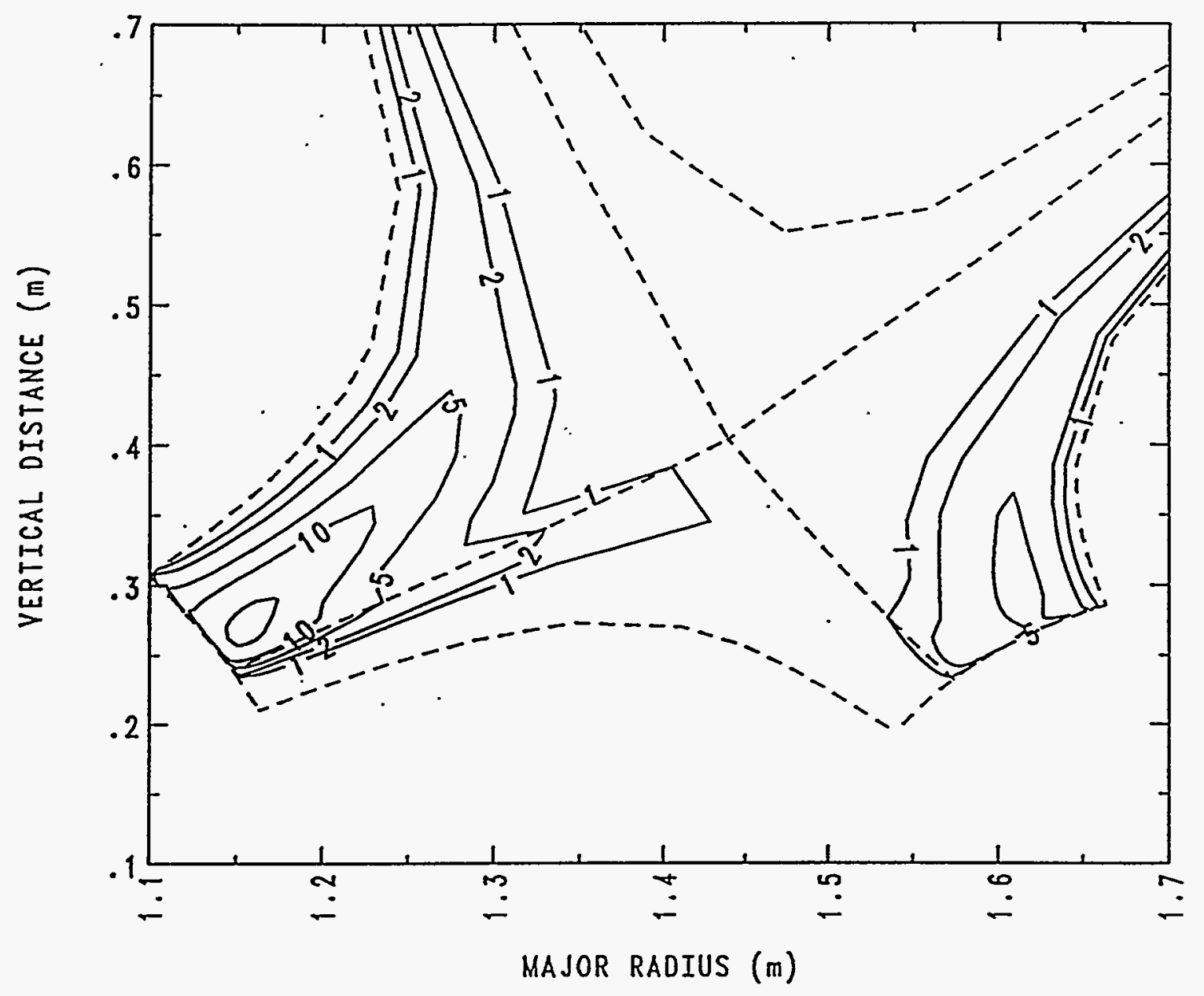

Fig. 5 